Research Article

J Exp Clin Med

2021; 38(3): 211-215

doi: $10.52142 /$ omujecm.38.3.1

\title{
Evaluation of morphology and viability of spheroid derived from Insulin-GLase cell line: A model system to understand Type 2 Diabetes Mellitus
}

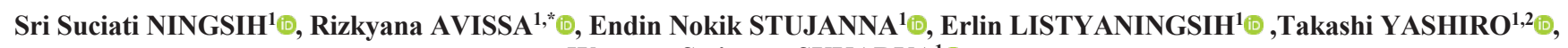 \\ Wawang Setiawan SUKARYA ${ }^{1}$ (i) \\ ${ }^{1}$ Faculty of Medicine, Universitas Muhammadiyah Prof. DR. HAMKA, Indonesia \\ ${ }^{2}$ Jichi Medical University School of Medicine, Japan
}

\begin{abstract}
\begin{tabular}{lllll}
\hline Received: 24.03 .2021 & $\bullet$ & Accepted/Published Online: 31.03 .2021 & Final Version: 23.04 .2021 \\
\hline
\end{tabular}
Abstract

Type 2 Diabetes Mellitus (T2DM) is one of the major health issues in the world. The cellular mechanism of T2DM is still not fully understood. It could be studied by using spheroid three-dimensional (3D) culture which is considered representative of the in vivo conditions. Several types of pancreatic $\beta$ cell lines have been used, one of which is the insulin-GLase (iGL) cell line. This study aims to evaluate the effect of cell density and incubation time on spheroid morphology and cell viability in order to understand which one can be considered as the best option in studying T2DM using iGL cell. Spheroid was made by using the Hanging drop method. The variations of initial seeding cells were 50, 100, 200, and 400 cells $/ \mu \mathrm{L}$ then incubated for $1,2,3$, and 4 days. The evaluated parameters in this study are spheroid morphology and cell viability. Spheroid morphology was observed by using inverted phase contrast microscope integrated with camera (Nikon) and NIS-Elements Analysis D software. Cell viability was determined by using LUNA-II ${ }^{\mathrm{TM}}$ Automated Cell Counter (Logos Biosystem). The result of this study showed that spheroid in all of the group cell concentration have formed since the first day and its diameter was significantly increased on the following days ( $<<0,05$ ). The spheroid size was positively correlated with the cell density in group 50-200 cells $/ \mu \mathrm{L}$. A single and stable spheroid morphology was observed in 50-100 cells $/ \mu \mathrm{L}$ group. Cell viability in $3 \mathrm{D}$ culture system was lower and significantly decreased since day 3 compared to $2 \mathrm{D}$ culture ( $\mathrm{p}<0.05 ; 0.01)$. In conclusion, spheroid derived from iGL cell line with a stable morphology and good viability could be obtained from a cell concentration of 50-100 cells / $\mu \mathrm{L}$ with two days of incubation.
\end{abstract}

Keywords: iGL, cell density, spheroid, viability

\section{Introduction}

Diabetes is a major health problem around the world. Corresponding to the International Diabetes Federation (IDF), the incidence of diabetes reached $9.3 \%$ or 460 million people of the world's total population in 2019. This number is expected to continuously increase in the next decade. Indonesia is the $7^{\text {th }}$ rank as the country with the highest number of diabetics patient in the world with a range of 10.7 million people. Type-2 diabetes (T2DM) is the type of diabetes with the largest number of patients (IDF Diabetes Atlas $9^{\text {th }}$ edition, 2019). T2DM is caused by inadequate insulin secretion and insulin resistance. Currently cellular and molecular mechanism of T2DM is not fully understood. However, nowadays it can be studied with by using in vitro model of pancreatic $\beta$ cell culture.

iGL cell line is a cell derived from the rat pancreatic $\beta$ cell sub-clone. These cells can express insulin-GLase in response to high environmental glucose levels. Human insulin protein were fused with Gaussia luciferase protein as a marker of insulin secretion (Suzuki et al., 2017). Another advantage of this cell is its ability to be cultured in 3D system. These cells will form an aggregate that gather and condense which is known as a spheroid. 3D culture as spheroid form is more identical to actual conditions of the in vivo microenvironment than monolayer culture by facilitating the interaction cell to cell and cell to extracellular matrix interaction (Ryu et al., 2019). Moreover, this culture system allows cells to grow from all directions in vitro conditions (Lee et al., 2019). This 3D spheroid culture model can be potentially used to study the cellular conditions and microenvironment of pancreatic $\beta$ cells and their correlation with the pathomechanism of T2DM. Furthermore, it could be used as an appropriate model for studying the activity and effectiveness of antidiabetic drugs. The spheroid model is a good model for testing the efficacy and toxicity of drugs, as proven in anti-tumor drugs research (Gong et al., 2015).

In this study, the hanging drop method was preferred because it is a relatively easy and simple method to generate the spheroids. It tends to be simple and does not require complicated tools and materials. This technique allows spheroids to form on the edge of the drop. The size of 
spheroid could be controlled by adjusting the volume of drop or the density of cell suspension (Bartosh and Ylostalo, 2014). In comparison to previous research, spheroids from iGL cells were obtained by 3D culture system that using specific plate, this technique is considered as easier and reproducible (Suzuki et al., 2017).

The limitation of the spheroid culture system is the greater the cell aggregate the more likely the cells in the spheroid center are deficient in nutrients and oxygen that causing cell death (Ryu et al., 2019). Therefore, this study aims to understand the effect of the initial cell density and incubation time on the morphology and cell viability of iGL cell spheroids. Furthermore, this is an original study to determine the most appropriate number of initial cell density and incubation time to generate spheroid derived from iGL cells with good shape and viability for advance further research.

\section{Materials and methods}

\section{1. iGL cell preparation and propagation}

iGL cells are derived from the rat pancreatic $\beta$ cells (Cosmo Bio, IGL01C, Japan). The cells thawed in the water bath for two minutes. Then administration of $1 \mathrm{~mL}$ of iGL medium culture (Cosmo Bio, IGLM, Japan) into the cryotube. Further, the cell was transferred directly to the $15 \mathrm{~mL}$ tube (Corning, 430791, USA) and add the medium until $10 \mathrm{~mL}$ volume. Next, it was centrifuged at a speed of $300 \mathrm{~g}$ for five minutes then discards supernatant. The cell pellet then washed with a $5 \mathrm{~mL}$ medium and centrifuged at a speed of $300 \mathrm{~g}$ for five minutes. The supernatant was discarded and resuspended with $1 \mathrm{~mL}$ medium and count the cells with an automatic cell counter (LUNA Automated Cell Counter, Logos Biosystem, South Korea). Seeding the $8 \times 10^{5}$ cells in $100 \times 20 \mathrm{~mm}$ dish (Corning, 430167, USA). Medium was replaced on the fourth day. After reached $70-90 \%$ of confluency, the cells were passaged.

Passage of the cell was performed by removing the remaining medium followed by washing with PBS (Gibco, 18912014, USA) once. Then $1 \mathrm{~mL}$ of Trypsin-EDTA $0.05 \%$ (Gibco, 25300054, USA) were added and incubate at $37^{\circ} \mathrm{C}$ for two minutes, subsequently. Next, $10 \mathrm{~mL}$ medium were added to deactivate the trypsin. The detached cells were transferred into the new tube, counted, and seeded to a new $100 \times 20 \mathrm{~mm}$ dish with $8-9 \times 10^{5}$ cells each dish afterward.

\subsection{D cell culture with hanging drop method}

After cell propagation, cells were collected and divided into four cell density variants which are 50,100, 200 and 400 cell $/ \mu \mathrm{L}$ cell suspension. The cell suspension drops were formed by placing $25 \mu \mathrm{L}$ cell suspension on the lid of a $100 \times 20 \mathrm{~mm}$ bacterial petri dish (SPL,10101, South Korea). The outer side of the dish lid were previously outlined with waterproof marker to make small squares approximately $5 \times 5$ $\mathrm{mm}$ in size before used, so the cell suspension drop could be placed in a relatively identical distance. 80 drops were prepared for each cell concentration which performed in two petri dishes. $20-25 \mathrm{~mL}$ if sterile PBS were added to the bottom part of the dish. Once the all the drop has completed, the lid was flipped quickly and carefully to maintain the drops. Then the lid was used to cover the bottom part of the dish that already contained PBS. The petri dishes were placed in the incubator with $37^{\circ} \mathrm{C}$ and $5 \%$ of $\mathrm{CO}_{2}$ for $1,2,3$, and 4 days.

\subsection{Spheroid morphology evaluation}

The study evaluated spheroid morphology by the size and shape of the spheroid from each variation of cell concentration during different time of incubation. The morphology was observed lusing inverted contrast-phase microscope (Nikon, Ts2 Ph Fl, Japan) integrated with camera (Nikon, DS-Fi3, Japan). The spheroid diameter was measured by using NIS-Elements Analysis D 5.20.00 64-bit software. Measurements were performed at 30 drops per treatment (15 drops per dish). When there was an excessive number of spheroids in one drop, the average size would be the final size. Measurements are performed on two orthogonal spheroid diameters ( 11 and $\mathrm{d} 2$ ) and then the estimation of diameter (D) is calculated by calculating the root of the multiplication of the two diameters $(\mathrm{D}=\sqrt{d 1 d 2})$ (Shi et al., 2018).

\subsection{Evaluation of cell viability}

Cell viability was analyzed by using trypan blue and LUNAII ${ }^{\mathrm{TM}}$ Automated Cell Counter (Logos Biosystem, South Korea). Cells from 2D and 3D cultures were harvested to produce cell suspense containing single cells. The spheroid was dispersed by using Trypsin-EDTA $0.05 \%$ (Gibco, 25300054, USA) and gentle pipetting. Afterwards, each cell suspension was mixed with $10 \mu \mathrm{L}$ Trypan Blue $0.4 \%$ with a ratio of $1: 1$. Then it was approximately homogenized for five seconds by using a $10 \mu \mathrm{L}$ micropipette (Appendorf, USA). next, $10 \mu \mathrm{L}$ mixed cells and trypan blue were removed into the counting slide. Finally, the slide was inserted into the device and the monitor displayed the result immediately after few minutes.

\subsection{Data analysis}

The data were analyzed using the STATA.12 software. Student's t-tests or two-way analysis of variance and Tukey's post hoc tests are used to determine significant differences between the groups.

\section{Results}

\subsection{Spheroid size and morphology}

The formation of cell spheroids began in all variation groups of cell density since the first day after cell seeding. Fig. 1 showed that the spheroids formed most closely resemble round or oval balls. This shape is found in spheroids derived from all variations except 200 cells $/ \mu \mathrm{L}$. In 200 cells $/ \mu \mathrm{L}$ variant, the spheroids were found in irregular shape.

The concentration of cells has positive correlation with the diameter of the spheroid. However, the positive correlation only occurred in the group of $50-200$ cells $/ \mu \mathrm{L}$. At a higher 
cell concentration, 400 cells $/ \mu \mathrm{L}$, the diameter of the spheroid $\quad$ is smaller but found in greater quantities.
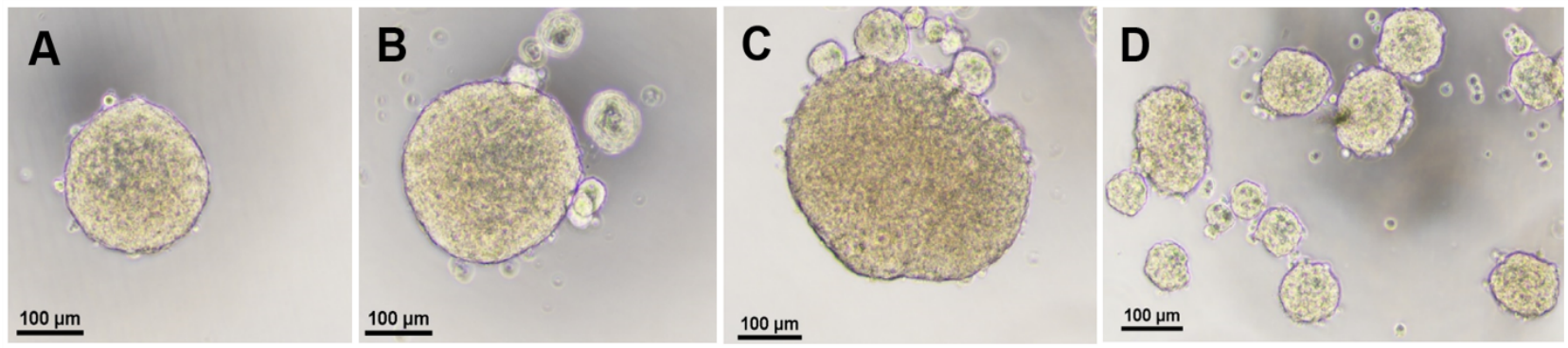

Fig. 1. Spheroid from 50 cells $/ \mu \mathrm{L}$ (A), 100 cells $/ \mu \mathrm{L}$ (B), 200 cells $/ \mu \mathrm{L}(\mathrm{C})$, and 400 cells $/ \mu \mathrm{L}$ (D) with two days of incubation (Phase contrast microscope, mag. 100x)

Discrepancies in spheroid size are observed from the first to the fourth day after are illustrated in Fig. 2. Spheroids become larger and more solid as the day went. Concentration and duration of incubation have a significant effect on the size of the spheroid. Moreover, there is a positive correlation between the diameter of the spheroid and the day of incubation.

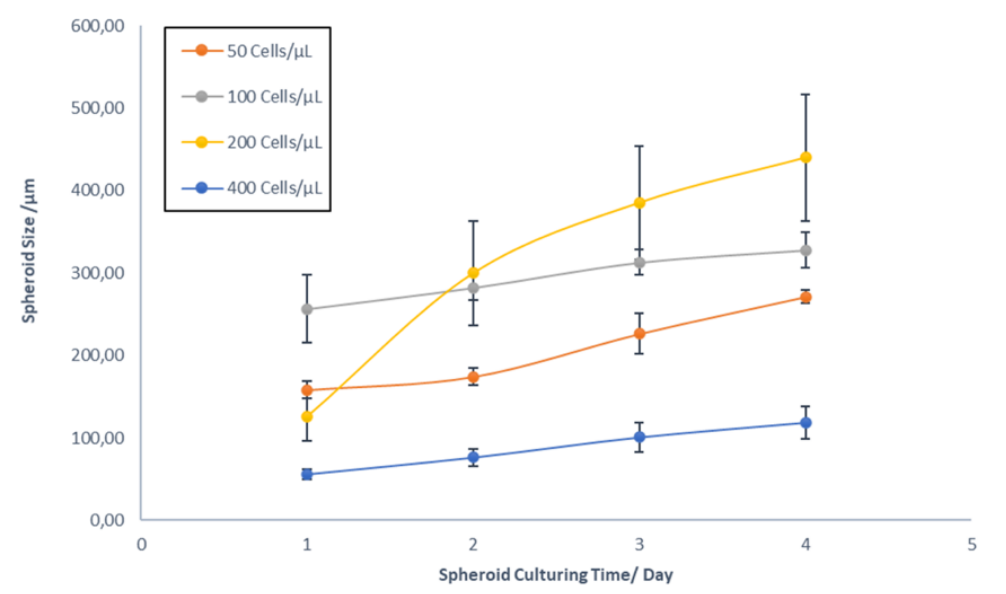

Fig. 2. Size comparison of spheroid from different initial cell seeding densities from day 1 to 4 . Data represent the mean \pm standard deviation (SD) of each experiment $(n=30)$. Data included was \pm 2 SD. Statistical analysis was performed by to way ANOVA ( $p=<0.01)$. There is strong positive correlation between spheroid and day of incubation (Pearson, $\mathrm{r}=0,4443$ )

\subsection{Spheroid cell viability}

The viability of iGL cells cultured in 3D culture system is relatively unstable than $2 \mathrm{D}$ monolayer culture. 2D cultures mostly maintain their viability in up to $80 \%$ for all groups of cell concentration until the fourth day. During two days of incubation, the 2D and 3D culture systems showed no significant differences for the cell viability in groups of 50,100 , and 200 cells/ $\mu \mathrm{L}$. However, the cell viability begins to fall significantly from the third to the fourth day in $3 \mathrm{D}$ cultures. Result is shown in Fig. 3.

\section{Discussion}

An understanding of the cellular mechanism of insulin secretion and its correlation to mechanism of T2DM can be studied with an in-vitro model of pancreatic $\beta$ cell culture. Several variants of widely used pancreatic $\beta$ cell line are RINm5F (Lai et al., 2014), EndoC-bH1 (Esguerra et al., 2020), INS-1 cells (Baidwan et al., 2017), and iGL (Suzuki et al., 2017). Most of the cells are potentially form 3D structure like spheroid. Spheroid has been counted as appropriate in vitro model for last decade due to it closely mimic the in vivo condition (Bartosh and Ylostalo, 2014). Several methods have been used for generating spheroids i.e., hanging drop, gel embedding, magnetic levitation, and spinner culture (Ryu et al., 2019). Some of these methods need scaffolds (Bresciani et al., 2019). Each of these methods has its own advantages and disadvantages respectively. Appropriate method of the study is selected by considering the research objectives and availability of tools and materials. This study employed hanging drop method due to it is an easy, simple and reproducible method.

This study proved that spheroids from iGL cells can be generated by using 3D culture system with Hanging Drop method as shown in Fig. 1. The size of the spheroid was affected by cell density and time of incubation. Fig. 2 showed that the size of the spheroid was positively correlated with the initial cell seeding number at concentrations of 50-200 cells $/ \mu \mathrm{L}$. This result relatively similar with the previous study by using a variety of cell lines with different cell number such as HIEC-6 (Flampouri et al., 2019), MCF-7 (Gong et al., 2015), HCT-116, UM-UC-3, and HeLa cells (Pereira et al., 2017). 

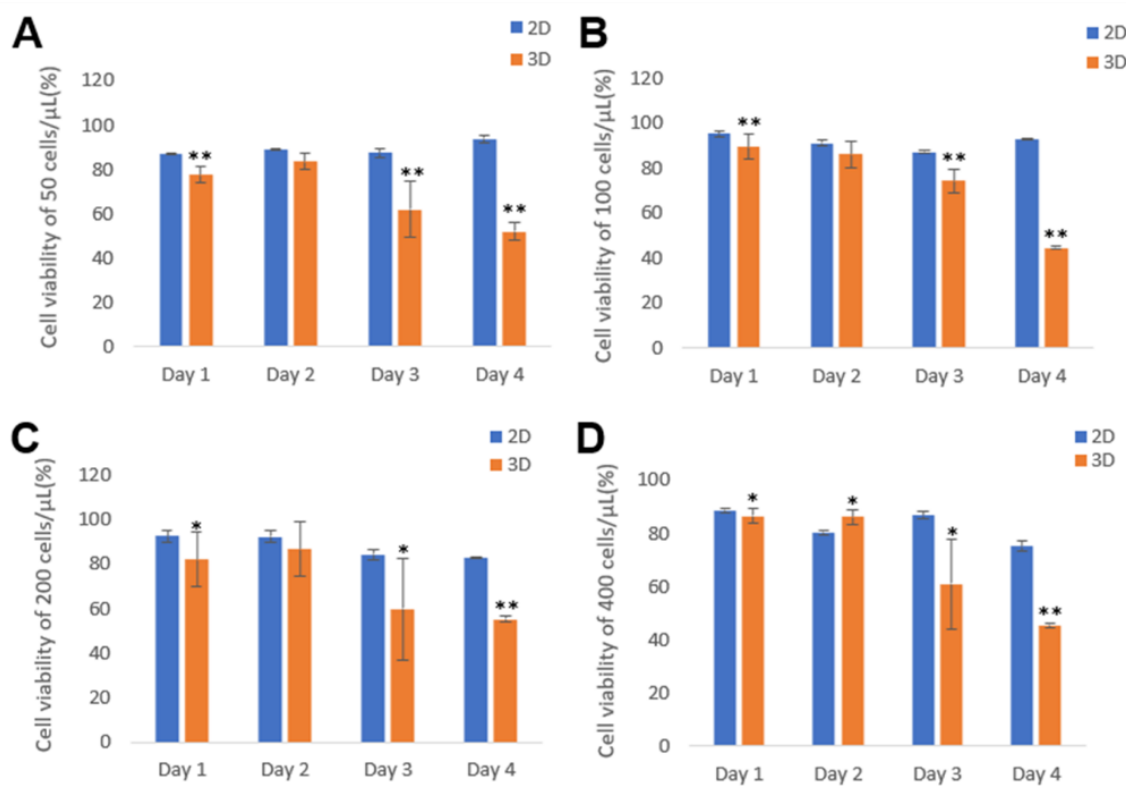

Fig. 3. Cell viability of iGL cells in 3D and $2 \mathrm{D}$ culture from 1-4 days of incubation with various cell densities (A-D 50,100,200, and 400 cells $/ \mu \mathrm{L}$ respectively). The differences between $2 \mathrm{D}$ and $3 \mathrm{D}$ were analyzed using student $\mathrm{t}$-test $(* * \mathrm{p}<0.01, \quad * \mathrm{p}<0.05)$

Fig. 1 illustrated that spheroid derived from iGL cell tends to be stable and consistent morphology in groups of 50 and 100 cells $/ \mu \mathrm{L}$ with a round or oval shape and mostly a single spheroid. On contrary, the group of 200 cells $/ \mu \mathrm{L}$ had an irregular shape and surrounded by numerous spheroids in smaller size. Different results were found in the cell group of 400 cells $/ \mu \mathrm{L}$. The size of the spheroids of this group tends to be smaller, even smaller than the group of 50 cells $/ \mu \mathrm{L}$. However, greater quantity of the spheroids was found in this groups, rather than other groups that had a single spheroid or multiple spheroids with one large dominant spheroid. It is apparently due to the differences in the microenvironment between groups. The formation of spheroids by cell aggregation is a complex process influenced by the surrounding micro-environment such as extracellular matrix, cell to cell junctions, and dissolved proteins in the medium. Additionally, the formation of spheroids is also determined by the phenotype of the cell type used (Repin et al., 2014). Each cell has a different fusion speed and model. Spheroids can be formed by the fusion of a single cell or cell aggregate thus forming a larger and denser cell aggregate to become a spheroid (Kosheleva et al., 2020).

Fig. 3 showed that the cell viability of spheroid from all groups of cell concentrations showed similar results to $2 \mathrm{D}$ monolayer culture in two-day incubation, except for the $400 / \mu \mathrm{L}$ group that tends to have higher viability. However, in general, viability of spheroid cells is most likely lower than $2 \mathrm{D}$ monolayer culture due to inequality in gaining oxygen and nutrition. Cells in 2D culture have better access to nutrients and oxygen than a spheroid. The spheroid consists of a multilayered mass of cells that form two zones, the middle and peripheral zones. Cells of the middle zone were lack of nutrients and oxygen so they tend to be in the quiescent phase or cell death either by apoptosis or necrosis. This zone looks darker on observations with a microscope inverted contrast phase (Zanoni et al., 2016). In this study, the dark zone was found in spheroids since the $2^{\text {nd }}$ day of incubation. However, it is still seemed as a small spot. The dark zones expanded from day 3 and almost cover all spheroids on day 5 (data not shown). From this result, we can conclude that iGL cell 3D culture with Hanging Drop method $(25 \mu \mathrm{L}$ per drop) allows conducive microenvironment conditions until the $2^{\text {nd }}$ day of incubation. The result relatively consistent with the data of cell viability. The viability of spheroid cells decreased intensely in $3 \mathrm{D}$ culture from day 3 of incubation compared to 2D monolayer culture. This result is consistent with a common fact about the increase of incubation time results in decreased nutrients and increased toxic metabolites for cells (Ryu et al., 2019). That was one of the disadvantages of Hanging Drop culture method is the difficulty of the medium replacement process (Bresciani et al., 2019).

Despites of the good results, there are some limitations of this study. First, iGL cells need a special medium and reagents that are relatively more expensive than widely used basic medium. Researchers still unable to maximize the potential of iGL cells which insulin secretion can be detected directly from the fluorescence under the fluorescent microscope. Therefore, further research is required to explore the potential of iGL cells as an in-vitro model to study DMT2 especially in terms of cellular mechanisms of insulin secretion.

In conclusion, we found that the initial cell density and time of incubation have a significant effect on spheroid morphology and cell viability. Spheroid derived from iGL cell line with a stable morphology and good viability could be obtained from a cell concentration of $50-100$ cells $/ \mu \mathrm{L}$ with two days incubation. 


\section{Conflict of interest}

There is no conflict of interests in this research.

\section{Acknowledgments}

The authors would like to thank Dr. Shino Ohtaka from Cosmo Bio for technical advices with the experiment.

\section{Funding}

This research was supported by a grant from Universitas Muhammadiyah Prof. DR. HAMKA 2019-2020. The funders had no role in study design, data collection and analysis, decision to publish, or preparation of the manuscript.

\section{References}

1. Baidwan, S., Chekuri, A., Hynds, D. A. L., Kowluru, A., 2017. Glucotoxicity promotes aberrant activation and mislocalization of Ras-related C3 botulinum toxin substrate 1 [Rac1] and metabolic dysfunction in pancreatic islet $\beta$-cells: reversal of such metabolic defects by metformin. Apoptosis. 22(11), 1380-1393.

2. Bartosh, T. J., Ylostalo, J. H., 2014. Preparation of antiinflammatory mesenchymal stem/precursor cells (MSCs) through sphere formation using hanging-drop culture technique. Curr. Protoc. Cell Biol. 1, (Suppl. 28).

3. Bresciani, G., Hofland, L. J., Dogan, F., Giamas, G., Gagliano, T., Zatelli, M. C., 2019. Evaluation of spheroid 3D culture methods to study a pancreatic neuroendocrine neoplasm cell line. Front. Endocrinol. 10, 1-10.

4. Esguerra, J. L. S., Ofori, J. K., Nagao, M., Shuto, Y., Karagiannopoulos, A., Fadista, J., Sugihara, H., Groop, L., Eliasson, L., 2020. Glucocorticoid induces human beta cell dysfunction by involving riborepressor GAS5 LincRNA. Mol. Metab. 32, 160-167.

5. Flampouri, E., Imar, S., Oconnell, K., Singh, B., 2019. Spheroid3D and monolayer-2D intestinal electrochemical biosensor for toxicity/viability testing: Applications in drug screening, Food Safety, and Environmental Pollutant Analysis. ACS Sensors. 4(3), 660-669.

6. Gong, X., Lin, C., Cheng, J., Su, J., Zhao, H., Liu, T., Wen, X., Zhao, P., 2015. Generation of multicellular tumor spheroids with microwell-based agarose scaffolds for drug testing. PLoS ONE. 10(6), 1-18.
7. IDF Diabetes Atlas 9th edition. 2019. IDF Diabetes Atlas 9th edition 2019. In International Diabetes Federation Diabetes Atlas, Ninth Edition.

8. Kosheleva, N. V., Efremov, Y. M., Shavkuta, B. S., Zurina, I. M., Zhang, D., Zhang, Y., Minaev, N. V., Gorkun, A. A., Wei, S., Shpichka, A. A., Saburina, I. N., Timashev, P. S., 2020. Cell spheroid fusion: Beyond liquid drops model. Sci. Rep. 10(1), 115.

9. Lai, X., Kang, X., Zeng, L., Li, J., Yang, Y., Liu, D., 2014. The protective effects and genetic pathways of thorn grape seeds oil against high glucose-induced apoptosis in pancreatic $\beta$-cells. BMC Complement. Altern. Med. 14, 1-7.

10. Lee, D., Pathak, S., Jeong, J. H., 2019. Design and manufacture of 3D cell culture plate for mass production of cell-spheroids. Sci. Rep. 9(1), 1-8.

11. Pereira, P. M. R., Berisha, N., Bhupathiraju, N. V. S. D. K., Fernandes, R., Tomé, J. P. C., Drain, C. M., 2017. Cancer cell spheroids are a better screen for the photodynamic efficiency of glycosylated photosensitizers. PLoS ONE. 12(5), 1-21.

12. Repin, V. S., Saburina, I. N., Kosheleva, N. V., Gorkun, A. A., Zurina, I. M., Kubatiev, A. A., 2014. 3D-Technology of the formation and maintenance of single dormant microspheres from 2000 human somatic cells and their reactivation in vitro. B. Exp. Biol. Med. 158(1), 137-144.

13. Ryu, N. E., Lee, S. H., Park, H., 2019. Spheroid culture system methods and applications for mesenchymal stem cells. Cells. $8(12), 1-13$.

14. Shi, W., Kwon, J., Huang, Y., Tan, J., Uhl, C. G., He, R., Zhou, C., Liu, Y., 2018. Facile tumor spheroids formation in large quantity with controllable size and high uniformity. Sci. Rep. $8(1), 1-9$.

15. Suzuki, T., Kanamori, T., Inouye, S., 2017. Quantitative visualization of synchronized insulin secretion from 3D-cultured cells. Biochem. Biophys. Res. Commun. 486(4), 886-892.

16. Zanoni, M., Piccinini, F., Arienti, C., Zamagni, A., Santi, S., Polico, R., Bevilacqua, A., Tesei, A., 2016. 3D tumor spheroid models for in vitro therapeutic screening: A systematic approach to enhance the biological relevance of data obtained. Sci. Rep. 6, $1-11$. 